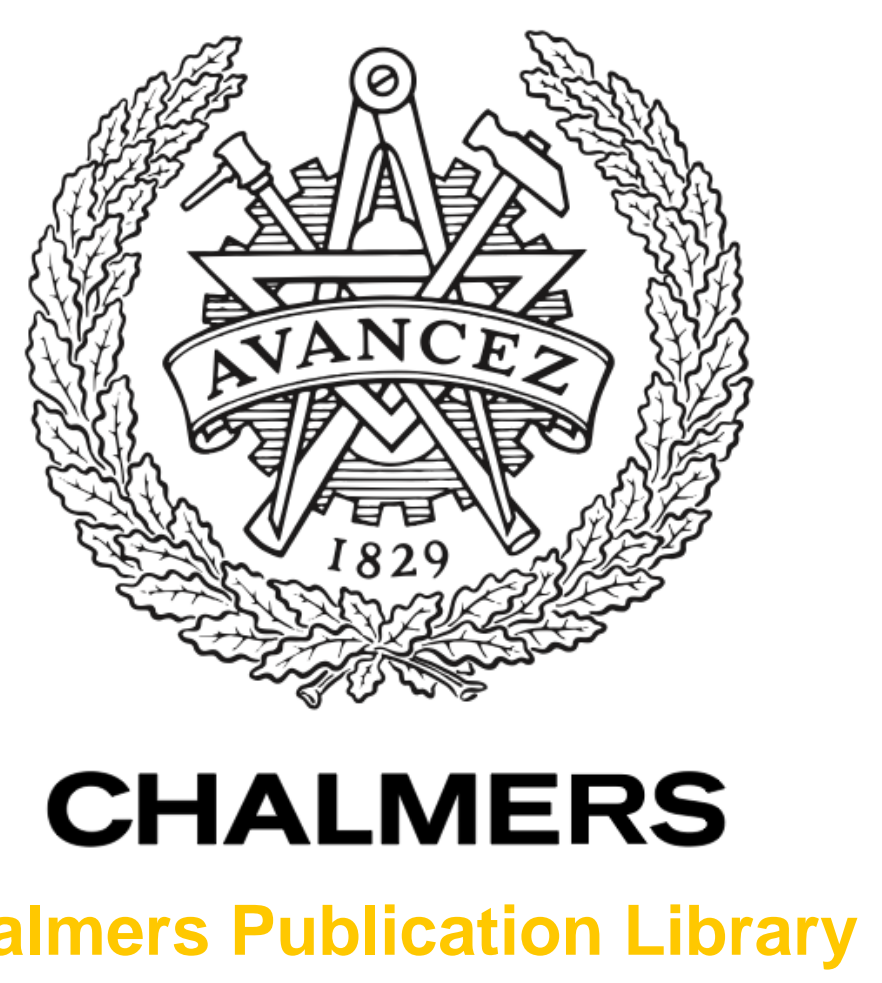

Chalmers Publication Library

Image Reconstruction in Microwave Tomography Using a Dielectric Debye Model

This document has been downloaded from Chalmers Publication Library (CPL). It is the author's version of a work that was accepted for publication in:

IEEE Transactions on Biomedical Engineering (ISSN: 0018-9294)

Citation for the published paper:

Fhager, A. ; Gustafsson, M. ; Nordbo, S. (2012) "Image Reconstruction in Microwave

Tomography Using a Dielectric Debye Model". IEEE Transactions on Biomedical

Engineering, vol. 59(1), pp. 156 - 166.

http://dx.doi.org/10.1109/TBME.2011.2168606

Downloaded from: http://publications.lib.chalmers.se/publication/153165

Notice: Changes introduced as a result of publishing processes such as copy-editing and formatting may not be reflected in this document. For a definitive version of this work, please refer to the published source. Please note that access to the published version might require a subscription. 


\title{
Image Reconstruction in Microwave Tomography Using a Dielectric Debye Model
}

\author{
Andreas Fhager, Member, IEEE, Mats Gustafsson, Member, IEEE, and Sven Nordebo,Senior Member, IEEE,
}

\begin{abstract}
In this paper quantitative dielectric image reconstruction based on broadband microwave measurements is investigated. A time domain based algorithm is derived where Debye model parameters are reconstructed in order to take into account the strong dispersive behavior found in biological tissue. The algorithm is tested with experimental and numerical data in order to verify the algorithm and to investigate improvements in the reconstructed image resulting from the improved description of the dielectric properties of the tissue when using broadband data. The comparison is made in relation to the more commonly used conductivity model. For the evaluation two examples were considered, the first was a lossy saline solution and the second was less lossy tap water. Both liquids are strongly dispersive and used as background medium in the imaging examples. The results show that the Debye model algorithm is of most importance in the tap water for a bandwidth of more than $1.5 \mathrm{GHz}$. Also the saline solution exhibits a dispersive behavior but since the losses restrict the useful bandwidth the Debye model is ofless significance even if somewhat larger and stronger artifacts can be seen in the conductivity model reconstructions.
\end{abstract}

Index Terms -Finite-difference time-domain (FDTD) methods, Debye model, dispersion, microwave imaging, microwave measurements.

\section{I NTRODUCTION}

$\mathrm{M}$ ICROWAVE imaging has the potential of solving some of the outstanding problems in today's health care. A large part of the global research effort in this area is focused on breast cancer detection which has reached a state where early clinical studies are being made and has been reported in the literature, [1]-[4]. Other biomedical areas, such as diagnostics of stroke and functional imaging of extremity soft tissue are also investigated. Furthermore microwave applications are researched for various applications such as ground surface mapping of the earth, detection of underground objects, nondestructive testing of materials, detection of defects and cracks in construction materials, etc.

A variety of different approaches to microwave imaging are under investigation and they can be divided into sub-classes in many different ways. For example single-frequency methods can be distinguished from multi-frequency and ultra-wideband

Manuscript received November 3, 2010; revised January 26, 2011. This work was supported in part by VINNOVA within the VINN Excellence Center Chase, and in part by SSF within the Strategic Research Center Charmant.

A. Fhager is with Biomedical Engineering Division, Department of Signal and Systems, Chalmers University of Technology, SE-41296 Goteborg, Sweden. (Email: andreas.fhager@chalmers.se).

M. Gustafsson is with the Department of Electrical and Information Technology, Lund University, SE-221 00 Lund, Sweden.

S. Nordebo is with the School of Mathematics and Systems Engineering Linnæus University, SE-351 95 V äxjö, Sweden. approaches. A different way to classify the methods is to separate microwave tomographic imaging from radar-based imaging. In microwave tomography the goal is to quantitatively reconstruct the dielectric parameters in the region under test whereas in a radar-based approach the goal is instead to generate an image of the relative scattering strength. Both these approaches are currently represented in clinical studies and examples from the literature are found in [1], [2] for the tomographic approach and in [3], [4] for the radar based approach. In light of this discussion, this paper is an example of a quantitative microwave tomography approach utilizing ultra-wideband data.

When turning from the single frequency approach to multifrequency or ultra-wideband techniques, dispersive effects are material properties ofincreasing importance for the modeling of electromagnetic wave propagation with increasing bandwidth. In principle all biological tissue types exhibit a dispersive pattern to some extent. It could for example be noticed in the microwave region already in some of the early dielectric measurements on healthy and malignant breast tissue, [5], [6]. It can also be clearly seen in the systematic characterization of human tissue published by Gabriel et al. in [7], [8]. In a more recent study on samples obtained from breast reduction and cancer surgery, primarily aiming at determining the contrast between malignant and different types of healthy tissues, the dispersive behavior is also evident, [9], [10]. It is clear that the widely used conductivity model, where the electromagnetic properties are described in terms of permittivity and conductivity, cannot be fitted to the data over any larger bandwidth. Instead different Cole-Cole and Debye models have been proposed for healthy and malignant breast tissues, [11]. Due to its computational efficiency the Debye model is particularly well suited for use in the FDTD computational scheme, [12].

The image reconstruction problem in microwave tomography is a specific example of an inverse scattering problem which is highly ill-posed and non-linear. To remedy the illposedness some kind of regularization scheme is usually used, such as the Tikhonov method. The regularization aims at enforcing bounds or constraints on the reconstructed solution and thus to reduce the number of degrees offreedom in the solution. It has been shown that the ill-posedness varies with factors such as the number and location of observations, the number offrequencies used, and the wavelength, [13]. In electrical impedance tomography (EIT) it has been concluded that the use of a dispersion model approach reduces the illposedness when multi-frequency data is available, [14], and it 
can be anticipated that this will be the case also in microwave tomography. Multi-frequency measurements with EIT has also been used to estimate dispersive Cole parameter data for lung edema detection, [15]. The dispersive tumor response was first considered in [16] for a pulsed microwave confocal system for breast cancer detection. In [17] the dispersive description of the breast tissue has been studied and the effects on the electromagnetic pulse propagation have been quantified. The results from this study on broad band applications indicated a need to model the dispersive properties in the image reconstruction procedure. This has also been made in some recently published works, [18], [19]. In both these publications a dispersion model has been adopted where the modeling parameters are estimated based on multi-frequency data and the algorithms are such that all frequencies are used simultaneously to form one image. In [18] a Gauss-Newton type of algorithm has been used for the optimization together with linear and logarithmic dispersion model and the algorithm in [19] is based on the distorted Born iterative method, [20] and a single pole Debye model. It is however well established that the two optimization methods are equivalent, [21], and the main difference between the two methods is thus the use of different dispersion models.

The non-linear property of the reconstruction problem also has to be considered in order to succeed with the reconstruction process without getting trapped in any local minimum and ending up with a sub-optimal or even incorrect reconstruction. One possibility to remedy the non-linearity, which is particularly useful when the reconstruction domain contains several objects with different sizes, is to utilize wideband spectral data. In general, the non-linearity of the inverse scattering problem is more significant with larger contrast, larger object size and higher frequency, [22]. On the other hand, it is desirable to use as high frequency content as possible to obtain a high resolution in the final image. To overcome the non-linearity while still benefiting from the improved resolution at higher frequencies a frequency-hopping approach has been suggested, [23]. It means that the image is first reconstructed with a low spectral content and once converged this image is taken as an initial guess to continued reconstruction at a higher frequency. This procedure can be repeated a number of times until the desired result have been achieved. Originally, this was proposed for use with a few single frequencies but we have implemented a time-domain version of this approach where pulses with increasing spectral content are used, [24]. A similar improvement as for the frequency-hopping approach can also be seen in the algorithms where several frequencies are used simultaneously, for example in [18]. We are however unaware of any comparative studies between the two approaches.

In conclusion, we see a number of potential benefits with a dispersive material model when utilizing wideband data for the imaging. In contrast to the previously published reconstruction algorithms where frequency domain formulations were used the purpose of this paper is to describe a reconstruction algorithm based on a time domain formulation and the Debye model. The performance of this algorithm is demonstrated with numerical and experimental data. Using the same algorithm we also investigate when a Debye model based algorithm can be used to improve the accuracy in the reconstructed image. The rest of the paper is organized as follows: In Section II the gradients are derived and a general description of the image reconstruction algorithm is given. Further in Section III the experimental imaging prototype is described together with the calibration procedure and algorithmic considerations specific to the imaging situation. In Section IV numerical and experimental data are used for imaging of an example in a lossy saline solution and an example in ordinary tap water, which in comparison to the saline solution is much less lossy. The results are compared with a conductivity model algorithm and related to the dispersive behavior of the dielectric properties. Finally in Section V the conclusions are presented.

\section{Debye Parameter Reconstruction Algorithm}

\section{A. Derivation of Gradients}

The underlying idea for image reconstruction is to compare measured transient scattering data with a simulated counterpart and to update the object in the simulation such that the computed data converges to the measured. This algorithm combines the FDTD solver for numerically modeling of the antenna system and for simulating the wave propagation between the antennas with an iterative conjugate gradient optimization method to solve the image reconstruction problem. A $2 \mathrm{D}$ version of this algorithm utilizing a conductivity model has been described in detail in the papers, [24], [25], and also a 3D version has been presented in [26]. In the conductivity model the dielectric properties are described by the permittivity, $\epsilon$, and the conductivity, $\sigma$. A slightly different version of the same algorithm has also been described in [27], and in [28][30] where reconstruction of the magnetic permeability also has been considered. The derivation of the gradients used for the Debye model parameter reconstruction is analogous to the derivation of the gradients used for recovering the conductivity model parameters. The derivation presented here closely follows the one given in [31] but differs in the way the electromagnetic sources are treated.

In the Debye model, the complex permittivity of the media is described as

$$
\epsilon(\mathbf{x}, \omega)=\epsilon_{\infty}(\mathbf{x})+\frac{\alpha(\mathbf{x})}{1+\mathrm{j} \omega \tau}+\frac{\sigma(\mathbf{x})}{\mathrm{j} \omega} .
$$

Considering real breast tissue there is a variation in the time constant $\tau$ for different tissue types, [11]. However the variation is such that the corresponding change in the complex permittivity is relatively small and probably of less importance in a real imaging situation. To first order approximation $\tau$ can most likely be assumed constant and it is therefore reasonable to assume that $\tau$ is a priori known and that the parameters to be reconstructed in the optimization process are $\epsilon_{\infty}(\mathbf{x})$, $\alpha(\mathbf{x})$ and $\sigma(\mathbf{x})$. The parameter $\alpha(\mathbf{x})$ can also be expressed in terms of the permittivity at the static, $\epsilon_{\text {static }}(\mathbf{x})$, and at the high frequency end, $\epsilon_{\infty}(\mathbf{x})$, of the frequency spectrum as $\alpha(\mathbf{x})=\epsilon_{\text {static }}(\mathbf{x})-\epsilon_{\infty}(\mathbf{x})$. In this paper we also discuss the conductivity model which could be obtained by setting $\alpha=0$ in (1) and thus the material is only described with the parameters $\epsilon_{\infty}(\mathbf{x})$ and $\sigma(\mathbf{x})$. However to avoid confusion 
with the notation we denote the conductivity model separately throughout the paper as

$$
\epsilon(\mathbf{x}, \omega)=\epsilon_{c}(\mathbf{x})+\frac{\sigma_{c}(\mathbf{x})}{\mathrm{j} \omega} .
$$

The time domain version of Maxwell's equations where the Debye model has been incorporated can now be expressed as

$$
\begin{aligned}
\nabla \times \mathbf{H} & =\epsilon_{\infty} \partial_{t} \mathbf{E}+\partial_{t} \mathbf{P}+\sigma \mathbf{E}+\mathbf{J}, \\
\nabla \times \mathbf{E} & =-\mu_{0} \partial_{t} \mathbf{H}, \\
\alpha \mathbf{E} & =\tau \partial_{t} \mathbf{P}+\mathbf{P} .
\end{aligned}
$$

Moreover we assume non-magnetic media such that $\mathbf{B}=\mu_{0} \mathbf{H}$ and that the initial conditions of the fields are $\mathbf{H}(t=0)=$ $\mathbf{E}(t=0)=\mathbf{P}(t=0)=\mathbf{0}$. The current $\mathbf{J}$ is used as the source in modeling the feeding of the antennas. This is also the complete set of equations to discretize and solve with the FDTD method.

The starting point for the optimization algorithm is the objective functional

$F\left(\alpha, \epsilon_{\infty}, \sigma\right)=\int_{0}^{T} \sum_{m=1}^{M} \sum_{n=1}^{N}\left|\mathbf{E}_{m n}\left(\alpha, \epsilon_{\infty}, \sigma, t\right)-\mathbf{E}_{m n}^{\mathrm{m}}(t)\right|^{2} \mathrm{~d} t$.

In this equation the parameters $\alpha, \epsilon_{\infty}$ and $\sigma$ are spatial distributions $\alpha(\mathbf{x}), \epsilon_{\infty}(\mathbf{x})$ and $\sigma(\mathbf{x})$ but for improved readability the spatial coordinates are suppressed. In the imaging process the spatial distribution of these parameters are also subject to optimization over the reconstruction domain in order to minimize the functional, (6). Furthermore $\mathbf{E}_{m n}\left(\alpha, \epsilon_{\infty}, \sigma, t\right)$ is the simulated field and $\mathbf{E}_{m n}^{\mathrm{m}}(t)$ is the corresponding measured data with antenna number $m$ used as transmitter and antenna $n$ as receiver. $M$ is the number of transmitters, $N$ is the number of receivers and $T$ is the duration of the pulse. For minimizing this objective functional we need gradients of type $\partial F / \partial \alpha, \partial F / \partial \epsilon_{\infty}$ and $\partial F / \partial \sigma$ with respect to the Debye model parameters in each grid point of interest. To obtain these gradients a first order perturbation analysis is performed where the parameters $\alpha, \epsilon_{\infty}$ and $\sigma$ are perturbed an amount $\delta$ in the directions $\alpha^{\prime}, \epsilon_{\infty}^{\prime}$ and $\sigma^{\prime}$ respectively: $\alpha \rightarrow \alpha+\delta \alpha^{\prime}$, $\epsilon_{\infty} \rightarrow \epsilon_{\infty}+\delta \epsilon_{\infty}^{\prime}$ and $\sigma \rightarrow \sigma+\delta \sigma^{\prime}$. To first order the fields are correspondingly perturbed $\delta \mathbf{H}^{\prime}, \delta \mathbf{E}^{\prime}$ and $\delta \mathbf{P}^{\prime}$ where the perturbation satisfies the set of equations derived from the original Maxwell's equations in (3)-(5),

$$
\begin{aligned}
\nabla \times \mathbf{H}^{\prime} & =-\epsilon_{\infty} \partial_{t} \mathbf{E}^{\prime}-\epsilon_{\infty}^{\prime} \partial_{t} \mathbf{E}-\sigma \mathbf{E}^{\prime}-\sigma^{\prime} \mathbf{E}-\partial_{t} \mathbf{P}^{\prime},(7) \\
\nabla \times \mathbf{E}^{\prime} & =-\mu_{0} \partial_{t} \mathbf{H}^{\prime}, \\
\alpha^{\prime} \mathbf{E} & =\tau \partial_{t} \mathbf{P}^{\prime}+\mathbf{P}^{\prime}-\alpha \mathbf{E}^{\prime} .
\end{aligned}
$$

The initial conditions of the perturbed fields must also satisfy $\mathbf{H}^{\prime}(t=0)=\mathbf{E}^{\prime}(t=0)=\mathbf{P}^{\prime}(t=0)=\mathbf{0}$. The corresponding expression of the perturbed objective functional in equation (6) with respect to the perturbations $\delta \alpha^{\prime}, \delta \epsilon_{\infty}^{\prime}$ and $\delta \sigma^{\prime}$ is to first order expressed as $F \rightarrow F+\delta F^{\prime}$ and it is straight forward to show

$$
\begin{aligned}
& F^{\prime}\left(\alpha, \epsilon_{\infty}, \sigma\right)= \\
& 2 \int_{0}^{T} \sum_{m=1}^{M} \sum_{n=1}^{N}\left[\mathbf{E}_{m n}\left(\alpha, \epsilon_{\infty}, \sigma, t\right)-\mathbf{E}_{m n}^{\mathrm{m}}(t)\right] \cdot \mathbf{E}_{m n}^{\prime} \mathrm{d} t .
\end{aligned}
$$

To obtain the gradients of the objective functional the adjoint fields $\tilde{\mathbf{E}}, \tilde{\mathbf{H}}, \tilde{\mathbf{P}}$ and $\tilde{\mathbf{J}}$ are introduced, which satisfy the adjoint Maxwell's equations

$$
\begin{aligned}
\nabla \times \tilde{\mathbf{H}} & =\epsilon_{\infty} \partial_{t} \tilde{\mathbf{E}}+\alpha \partial_{t} \tilde{\mathbf{P}}-\sigma \tilde{\mathbf{E}}-\tilde{\mathbf{J}} \\
\nabla \times \tilde{\mathbf{E}} & =-\mu_{0} \partial_{t} \tilde{\mathbf{H}} \\
\tilde{\mathbf{E}} & =-\tau \partial_{t} \tilde{\mathbf{P}}+\tilde{\mathbf{P}}
\end{aligned}
$$

The idea to solve the inverse problem in the time-domain with the help of adjoint fields was first introduced for a seismic problem, [32]. The adjoint problem has to be solved backwards in time from $t=T$ to $t=0$ with the initial conditions $\tilde{\mathbf{H}}(t=T)=\tilde{\mathbf{E}}(t=T)=\tilde{\mathbf{P}}(t=T)=\tilde{\mathbf{J}}(t=T)=0$. The adjoint problem is also associated with a forward problem where antenna $m$ was used as transmitter. In the adjoint problem the corresponding receivers, $n$, instead become the transmitting sources with the source term $\tilde{\mathbf{J}}$ set to the difference between the measured and the simulated fields as

$$
\tilde{\mathbf{J}}_{m n}=\sigma_{\text {eqiv }}\left[\mathbf{E}_{m n}\left(\alpha, \epsilon_{\infty}, \sigma, t\right)-\mathbf{E}_{m n}^{\mathrm{m}}(t)\right] .
$$

The constant $\sigma_{\text {eqiv }}$ is an arbitrary parameter and used to make the units equal. It is chosen to be $\sigma_{\text {eqiv }}=1 \mathrm{~S} / \mathrm{m}$. The adjoint fields in equations (11)-(13) are then cross multiplied with the perturbed equations (7)-(9) and integrated over time $[0, T]$ and the volume of interest for the reconstruction, $V$. At last the result is summed over all transmitters. For the source terms the integration over space is equivalent to a sum over all receivers and gives

$$
\begin{gathered}
F^{\prime}\left(\alpha, \epsilon_{\infty}, \sigma\right) \\
=2 \int_{0}^{T} \sum_{m=1}^{M} \sum_{n=1}^{N}\left[\mathbf{E}_{m n}\left(\alpha, \epsilon_{\infty}, \sigma, t\right)-\mathbf{E}_{m n}^{\mathrm{m}}(t)\right] \cdot \mathbf{E}_{m n}^{\prime} \mathrm{d} t \\
=2 \int_{V} \epsilon_{\infty}^{\prime} \sum_{m=1}^{M} \int_{0}^{T} \partial_{t} \mathbf{E} \cdot \tilde{\mathbf{E}} \mathrm{d} t \mathrm{~d} V \\
-2 \int_{V} \alpha^{\prime} \sum_{m=1}^{M} \int_{0}^{T} \mathbf{E} \cdot \partial_{t} \tilde{\mathbf{P}} \mathrm{d} t \mathrm{~d} V \\
\quad+2 \int_{V} \sigma^{\prime} \sum_{m=1}^{M} \int_{0}^{T} \mathbf{E} \cdot \tilde{\mathbf{E}} \mathrm{d} t \mathrm{~d} V
\end{gathered}
$$

With the inner product defined as $\langle x, y\rangle=\int_{V} x \cdot y \mathrm{~d} V$ and by writing the right hand side of (15) as $\left\langle G_{\epsilon_{\infty} /\left\langle\epsilon_{\infty}\right\rangle}, \epsilon_{\infty}^{\prime} /\left\langle\epsilon_{\infty}\right\rangle\right\rangle+$ $\left\langle G_{\alpha /\langle\alpha\rangle}, \alpha^{\prime} /\langle\alpha\rangle\right\rangle+\left\langle G_{\sigma /\langle\sigma\rangle}, \sigma^{\prime} /\langle\sigma\rangle\right\rangle$ the gradients can be identified as

$$
\begin{aligned}
G_{\epsilon_{\infty} /\left\langle\epsilon_{\infty}\right\rangle} & =2\left\langle\epsilon_{\infty}\right\rangle \sum_{m=1}^{M} \int_{0}^{T} \partial_{t} \mathbf{E}(\mathbf{x}, t, m) \cdot \tilde{\mathbf{E}}(\mathbf{x}, t, m) \mathrm{d} t \\
G_{\alpha /\langle\alpha\rangle} & =-2\langle\alpha\rangle \sum_{m=1}^{M} \int_{0}^{T} \mathbf{E}(\mathbf{x}, t, m) \cdot \partial_{t} \tilde{\mathbf{P}}(\mathbf{x}, t, m) \mathrm{d} t \\
G_{\sigma /\langle\sigma\rangle} & =2\langle\sigma\rangle \sum_{m=1}^{M} \int_{0}^{T} \mathbf{E}(\mathbf{x}, t, m) \cdot \tilde{\mathbf{E}}(\mathbf{x}, t, m) \mathrm{d} t .
\end{aligned}
$$


Here we also introduce a gradient scaling in form of the parameters $\left\langle\epsilon_{\infty}\right\rangle,\langle\sigma\rangle$, and $\langle\alpha\rangle$ to compensate for the imbalance between the sensitivity of the parameters. Finally the gradients in (16)-(18) are used with the conjugate-gradient method together with a successive parabolic interpolation line search to find the optimal step length, $\lambda^{i}$ for each iteration $i$ and the Debye model parameters are updated according to

$$
\begin{aligned}
\epsilon_{\infty}^{i+1}(\mathbf{x}) & =\epsilon_{\infty}^{i}(\mathbf{x})-\lambda^{i} G_{\epsilon_{\infty} /\left\langle\epsilon_{\infty}\right\rangle}^{i}(\mathbf{x}), \\
\alpha^{i+1}(\mathbf{x}) & =\alpha^{i}(\mathbf{x})-\lambda^{i} G_{\alpha /\langle\alpha\rangle}^{i}(\mathbf{x}), \\
\sigma^{i+1}(\mathbf{x}) & =\sigma^{i}(\mathbf{x})-\lambda^{i} G_{\sigma /\langle\sigma\rangle}^{i}(\mathbf{x}) .
\end{aligned}
$$

The reconstruction procedure is then iterated with the objective functional as a measure to monitor the convergence and to determine when the reconstruction is completed.

\section{B. Parameter Scaling}

A Fisher information based parameter scaling for microwave tomography is analyzed in [33], [34]. This generalizes the parameter scaling in [28] to include a radial scaling that improves the convergence in lossy media. In [33], [34], the Fisher information matrix with respect to the background medium was estimated using a two-dimensional analytical model of the antenna system. The results show that the sensitivity in the objective functional to a parameter update gradually decreases closer to the center of the imaging domain. As a consequence, the inversion is less sensitive to objects closer to the center. This effect is particularly pronounced in lossy media and needs to be compensated for to ensure optimal reconstruction of the targets. Based on the diagonal terms of the Fisher information matrix, a radial scaling of the gradients is deduced in which the parameter sensitivity is equalized in the entire imaging domain. The diagonal Fisher information elements are calculated as,

$$
\begin{aligned}
I_{\theta}(\rho)=C & \int k^{4}\left|P_{\theta}\left(k c_{0}\right)\right|^{2} \\
& \sum_{m=-\infty}^{\infty} \sum_{n=-\infty}^{\infty}\left|J_{m}(\sqrt{\epsilon} k \rho)\right|^{2}\left|J_{n}(\sqrt{\epsilon} k \rho)\right|^{2} \\
& \left|H_{m}^{(2)}(\sqrt{\epsilon} k b)\right|^{2}\left|H_{n}^{(2)}(\sqrt{\epsilon} k b)\right|^{2} \mathrm{~d} k,
\end{aligned}
$$

where $C$ is a constant, $\rho$ the radial coordinate, $k=\omega / c_{0}$ the wavenumber, $k_{\mathrm{c}}$ the center wavenumber, $J_{m}$ and $H_{m}^{(2)}$ are the Bessel function and the Hankel function of the second kind, respectively, both of order $m$. Further $\epsilon$ is the complex permittivity of the background from (1) and $b$ is the radius of the antenna array, $\theta$ represents the different parameters subject to estimation and $P_{\theta}(\nu)$ is defined as $P_{\epsilon_{\infty}}(\omega)=1, P_{\alpha}(\omega)=1 /(1+\mathrm{j} \omega \tau)$ and $P_{\sigma}=1 / \mathrm{j} \omega$. According to the Fisher information theory [33], [34], the integration should also be weighted with the spectral density of the electromagnetic pulse. In this paper, we approximate this with integration over the FWHM bandwidth assuming a uniform spectral density. It can be shown [33], [34] that to equalize the parameter sensitivity, the gradients in (16)-(18) should be scaled according to

$$
\langle\theta\rangle=\sqrt{I_{\theta}(\rho)} \text { for } \theta=\left\{\epsilon_{\infty}, \alpha, \sigma\right\} .
$$

\section{FDTD Modeling}

The numerical solution of Maxwell's equations, (3)-(5) and the adjoint problem in (11)-(13) is made with the FDTD algorithm, [12]. Our experimental antenna system consists of monopole antennas mounted above a ground plane. We use a 3D FDTD model of the antenna system with the thinwire approximation to model the monopoles, [35], and the resistive voltage source (RVS) with $50 \Omega$ impedance to model the feed at the transmitting, receiving and inactive antennas, [36], [37]. The ground plane is modeled as a perfect electric conductor, i.e. the corresponding tangential field components in the FDTD grid are set to zero. In a 2D version of the algorithm it is not possible to create a realistic antenna model but the transmitter is modeled with a hard point source, in which the field strength is prescribed at the source position. At the receiver locations the field values are sampled directly from the corresponding E-field component in the grid. The FDTD grid is terminated with the CPML absorbing boundary condition. This 2D configuration has also been used in this paper for generating synthetic scattering data for the image reconstruction examples.

\section{EXPERIMENTAL SYSTEM}

In the experimental prototype the measurement strategy is to measure the multistatic scattering matrix at a large number of frequencies and to use that data to generate a timedomain pulse via an inverse Fourier transformation. In the experimental system 20 monopole antennas, each of length $19.5 \mathrm{~mm}$ and diameter $0.8 \mathrm{~mm}$, are arranged evenly distributed on a circle with radius $100 \mathrm{~mm}$. The circle of antennas is centered on a square ground plane with side length $250 \mathrm{~mm}$ mounted at the bottom of a tank, made of $1 \mathrm{~cm}$ thick perspex sheets with inner measures $350 \times 350 \mathrm{~mm}^{2}$. To measure the multistatic matrix each antenna is operated as a transmitter as well as a receiver. The microwave measurements are conducted with network analyzer Agilent E8362 B PNA which is a two port network analyzer. To fully control the experiment a 2:32 switch multiplexer module, Cytec CXM/128-S-W, is used to automatically connect and disconnect the different combinations of antenna pairs to the network analyzer. Fig. 1 shows a photograph of the antenna array.

\section{A. Calibration of Measured Data}

As already mentioned the solution of the inverse problem heavily relies on the comparison between the measured and the simulated scattering data. In practice it is impossible to create an antenna model without modeling errors resulting in discrepancies between the measurements and the simulations of the system. To account for this a calibration procedure of the measured data is adopted such that

$$
E_{\text {calibrated }}^{\text {measured }}(\omega)=\frac{S_{\text {object }}^{\text {measured }}(\omega)}{S_{\text {referencence }}^{\text {meared }}(\omega)} E_{\text {reference }}^{\text {simulated }}(\omega),
$$

where $S_{\text {object }}^{\text {measured }}$ is the measured reflection and transmission coefficients of the test object, $S_{\text {reference }}^{\text {measured }}(\omega)$ is a reference measurement of an empty system, and $E_{\text {reference }}^{\text {simulated }}(\omega)$ is a corresponding reference simulation. Finally $E_{\text {calibrated }}^{\text {measured }}(\omega)$ is the 


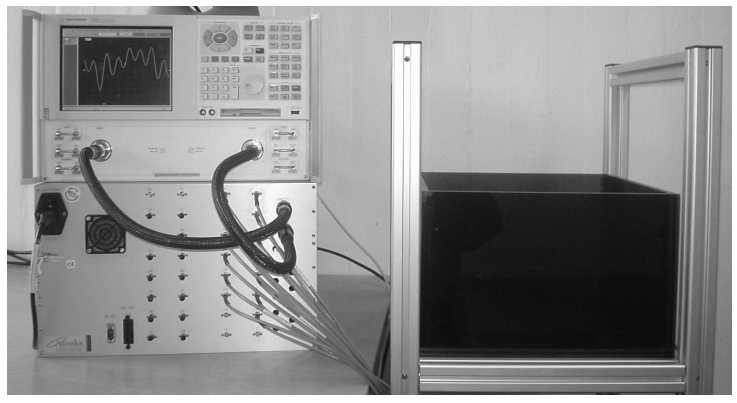

(a) The measurement system

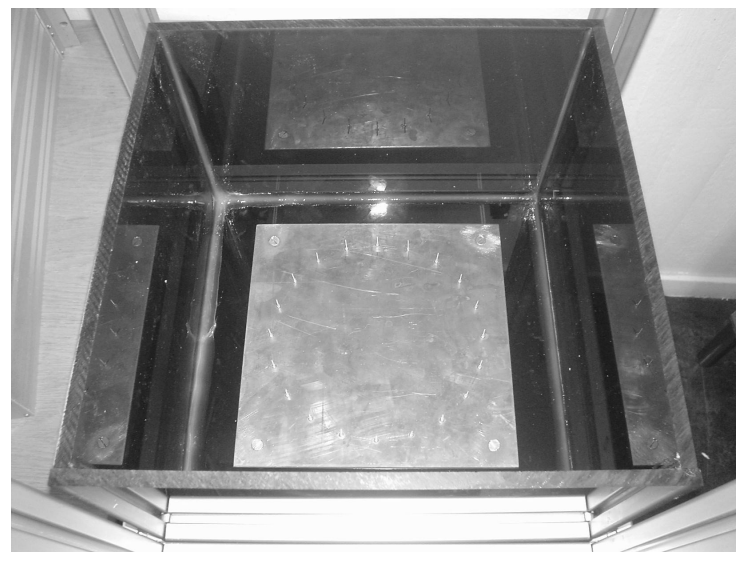

(b) The antenna array

Fig. 1. (a) Photograph of the measurement system. (b) Closeup of the antenna array placed inside a tank. The monopoles are seen mounted in a circle over the ground plane. The entire antenna system is mounted inside a tank made of perspex sheets.

calibrated data used for comparing with the FDTD simulations in the reconstruction process. The calibration is made here represented in frequency domain, for the simple reason that the measurements are made in network analyzer. This also means that $E_{\text {calibrated }}^{\text {measured }}(\omega)$ is the Fourier transformation of the simulated reference signal and an inverse transformation of the result is needed before it is used for comparison in the timedomain reconstruction algorithm. A more detailed description of the calibration procedure can be found in [25].

\section{B. Pseudo-3D Reconstruction}

In our experimental prototype the antennas are positioned in circular array on a plane as seen in Fig 1 (b). The possibility to accurately reconstruct out of plane objects is thus very limited: to do so it would be necessary also to make additional measurements outside the antenna plane. To allow imaging with the 3D algorithm of a test object with finite height we implemented a heuristic pseudo-3D technique that assumed constant properties of the test object as a function of height, $z$, above the ground plane. This results in an algorithm similar to [38] where the electromagnetic modeling is made in $3 \mathrm{D}$ and the dielectric parameter are recovered on a 2D grid. Considering imaging of a cylindrical target of finite height placed on the ground plane of the prototype one realizes that a 2D approximation of the cylinder is most accurate closest to the ground plane. In our work we therefore computed the gradient in the grid cell plane immediately above the ground plane and copied it upwards to the height of the test object. Consequently this method needs a priori information about the height of the reconstructed target.

Since the reconstruction problem is both non-linear and illposed the resulting image strongly depends on the adopted regularization technique, the initialization of the reconstruction and also the spectral content of the pulse, [24]. In the following examples we started the reconstruction procedure from the background dielectric values inside the antenna array. We also present reconstruction results using electromagnetic pulses with a spectral content that produced the most accurate reconstructed image.

\section{RESUlts}

In this section we discuss the differences in the electromagnetic modeling for the Debye model and the conductivity model. We also show reconstructed results originating from experimental and numerical data in order to test the algorithm and to investigate how the Debye model can improve the result over the conductivity model. Two examples were examined in this study, a lossy saline solution and less lossy ordinary tap water. To simplify the notation in the rest of the paper relative units will be used for $\epsilon_{\infty}, \epsilon_{\text {static }}$ and $\epsilon_{c}$ while SI units will be retained for $\sigma$.

\section{A. Modeling and reconstructions in a saline solution from experimental data}

In this section we use experimental measured data to verify and investigate the Debye model reconstruction algorithm. For the simulation of the experimental antenna array in the reconstruction algorithm we used a 3D FDTD model according to section II-C and the reconstruction problem was solved with the pseudo-3D version of the algorithm, section III-B. A mixture of water and $0.39 \%$ salt was used to create both a highly dispersive and a lossy background medium which was filled in the measurement tank up to a level of $60 \mathrm{~mm}$ above the ground plane. In Fig. 2 the real and the imaginary parts of the measured dielectric properties have been plotted together with fitted Debye and conductivity model data. The least-squares fitted Debye model is described according to (1) with parameters $\epsilon_{\infty}=38.1, \epsilon_{\text {static }}=78.0$ (or $\alpha=39.9$ ), $\tau=16.7 \mathrm{ps}$, and $\sigma=0.76 \mathrm{~S} / \mathrm{m}$. The corresponding best fit for the conductivity model was found to be $\epsilon_{c}=77.6$ and $\sigma_{c}=0.93 \mathrm{~S} / \mathrm{m}$. It can be seen that the Debye model describes the measured data very accurately. Comparing with the conductivity model the real part is also well described but there is a clear deviation in the imaginary part. When using the two different models in forward FDTD simulations the difference in the dielectric models will give rise to a corresponding difference in the computed scattering data. In order to illustrate the corresponding electromagnetic propagation scattering parameters were computed for the two models with the FDTD algorithm with grid size $1 \mathrm{~mm}$.

In Fig. 3 the magnitude and phase of the transmission coefficients, $S_{21}$ and $S_{91}$, have been plotted. Corresponding measured data for the saline solution is also shown. With the antennas numbered in consecutive order around the circle 


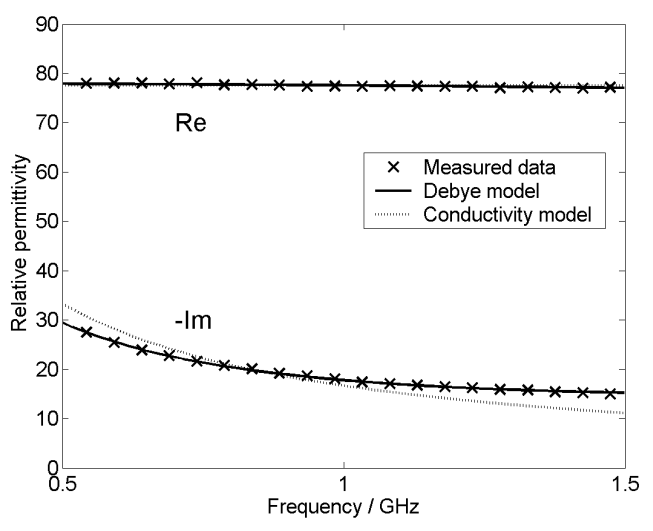

Fig. 2. This plot shows measured values of the dielectric properties for the saline solution used an background medium. Together with this are also the least-squares fitted Debye and conductivity models plotted.

$\mathrm{S}_{21}$ represents the transmission between two adjacent antennas and $S_{91}$ two opposing antennas. It can be seen that the agreement between the measured data and the simulated data is not perfect and also it varies between the two cases and with frequency. The use of a calibration procedure is an attempt to compensate for this discrepancy which is caused by imperfections in the numerical modeling in comparison to the actual prototype. We also note that for frequencies higher than about $1.2 \mathrm{GHz}$ the magnitude of the transmitted $S_{91}$ signal in the Debye model data goes down below $-80 \mathrm{~dB}$, a point where it in practice becomes very challenging to measure as the signals go below the noise floor of the measurement system. This is also seen in the measured data. This also means that only frequencies up to about $1.2 \mathrm{GHz}$ could be used for imaging.

In order to verify that the Debye parameter reconstruction algorithm works as expected we immersed two plastic rods with diameter $15 \mathrm{~mm}$ as targets in the lossy liquid, made the scattering measurements and reconstructed the corresponding images. One rod was placed in the center of the circular antenna array and one in a position halfway between the antennas and the center. Plastic is non-lossy with a relative permittivity $\epsilon_{c} \approx 2-3$, expressed in terms of the conductivity model, and consequently served as a high contrast target to the background. For the reconstruction we used the pseudo$3 \mathrm{D}$ formulation of the algorithm. To speed up the convergence we performed five iterations on a coarse grid of size $(4 \times 4 \times 4) \mathrm{mm}^{3}$. With the result from the coarse grid as a starting point on a $(2 \times 2 \times 2) \mathrm{mm}^{3}$ grid 20 additional iterations were performed. The center frequency was $f_{c}=1.0$ $\mathrm{GHz}$ and FWHM bandwidth $0.5 \mathrm{GHz}$. The reconstruction was started from a homogeneous background medium and no other regularization was used except that it is required that all reconstructed parameters should have a lower dielectric value than the background. In Fig. 4 the reconstructed results are shown both for the Debye model algorithm as well as for a reconstruction based on the conductivity model. In this figure the color scales have been varied such that the correct background values appear white. The dielectric background parameters have been taken from the data in Fig. 2. Further,

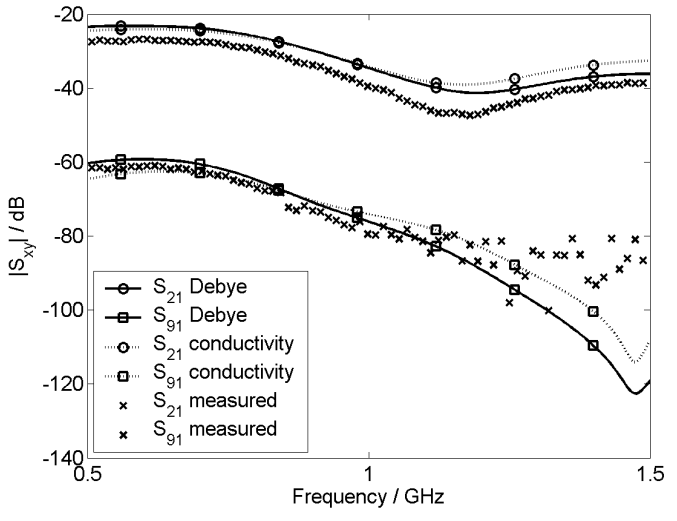

(a) Magnitude of $S_{21}$ and $S_{91}$

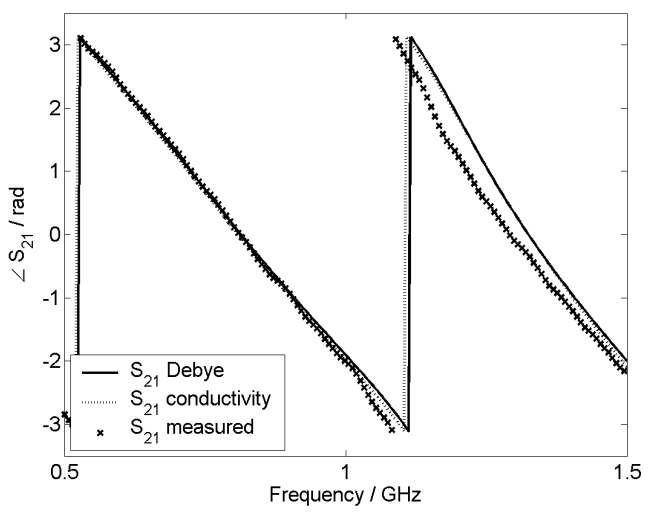

(b) Phase of $S_{21}$

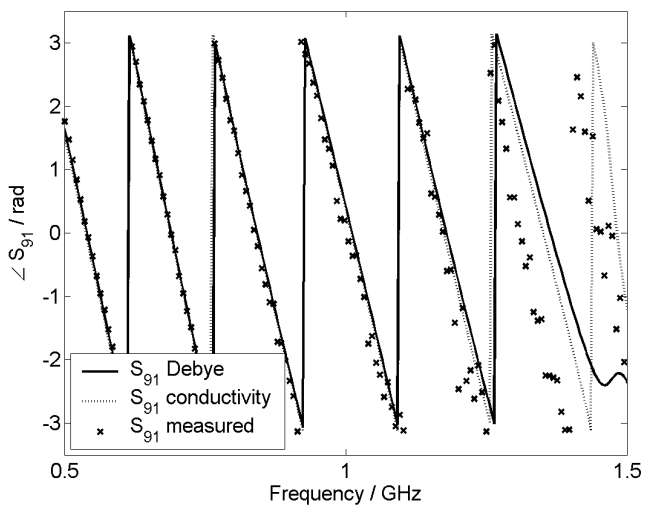

(c) Phase of $S_{91}$

Fig. 3. Magnitude and phase of the computed scattering data. (a) The magnitude of transmission coefficients, $S_{21}$ and $S_{91}$, have been plotted. The solid lines represent the result from the Debye model and the dotted lines the conductivity model. (b) and (c) The phase of the transmission coefficients.

the reconstruction domain has been marked with the large dashed circle and the original size and location of the targets have been marked with circles in the images. In general the targets have been reconstructed in the correct positions and with appropriate sizes, however with dielectric properties that are not quite reaching the expected values. Given the small difference between the two background models within the signal bandwidth it is also quite natural that the corresponding reconstructions are very similar. However there is a slight ten- 


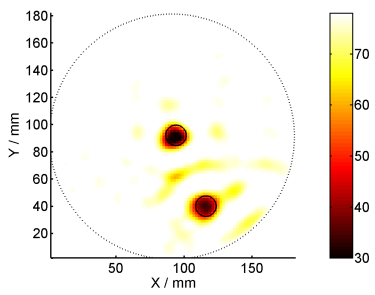

(a) Debye model, $\epsilon_{\text {static }}$

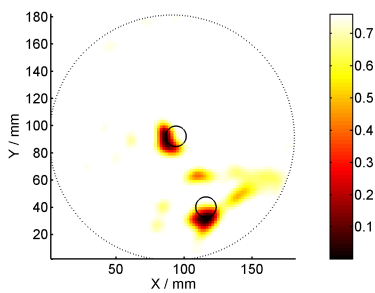

(c) Debye model, $\sigma$ in $\mathrm{S} / \mathrm{m}$

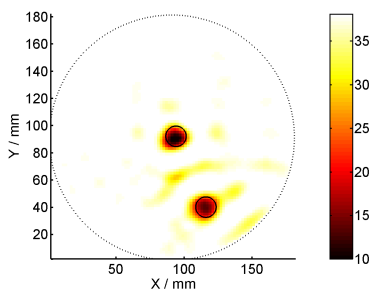

(e) Debye model, $\epsilon_{\infty}$

Fig. 4. Reconstructions of the two plastic targets immersed in a lossy liquid. The correct size and position of the targets are shown with the circles. Reconstructions with both the Debye model and the conductivity models are shown.

dency to more and larger artifacts in the reconstructions made with the conductivity model algorithm which could possibly be related to the larger errors caused by the conductivity model.

\section{B. Reconstruction of targets in pure water}

In the previous example the reconstruction was made over only $500 \mathrm{MHz}$ bandwidth since the losses of the saline solution prevents the use of higher frequency components than about $1.2 \mathrm{GHz}$. In this section we present a $2 \mathrm{D}$ imaging example based on numerically simulated data to investigate how the dielectric modeling and the bandwidth affects the reconstruction of targets immersed in ordinary tap water. The comparison is made such that forward scattering data is generated with the Debye model in order to simulate measurements. Later the reconstruction is made using both the Debye model algorithm and the conductivity model algorithm. The properties of the targets were close to what was found in [9] for healthy breast tissue rich in fibroconnective and/or glandular tissue, $\epsilon_{\text {static }}=39.8, \epsilon_{\infty}=7.82, \tau=10.24 \mathrm{ps}$ and $\sigma=0.71 \mathrm{~S} / \mathrm{m}$. The parameters of water was determined by fitting a Debye model to measured dielectric values and found to be $\epsilon_{\text {static }}=77.5, \epsilon_{\infty}=4.55, \sigma=0.0 \mathrm{~S} / \mathrm{m}$ and $\tau=8.1 \mathrm{ps}$. For the targets the values were modified slightly to $\epsilon_{\text {static }}=40.0, \epsilon_{\infty}=4.55$ and $\tau=8.1 \mathrm{ps}$, thus the values of $\epsilon_{\infty}$ and $\tau$ are equal to those of water and $\sigma$ equal to zero. This was made in order to reduce the complexity of the reconstruction scenario. A plot of measured dielectric data for water and the corresponding fitted Debye model is shown in Fig. 5. In the same figure is plotted a conductivity model which was determined from the real and imaginary parts of the Debye model at $0.5 \mathrm{GHz}$. It was found to be $\epsilon_{c}=77.5$ and $\sigma_{c}=0.05 \mathrm{~S} / \mathrm{m}$.

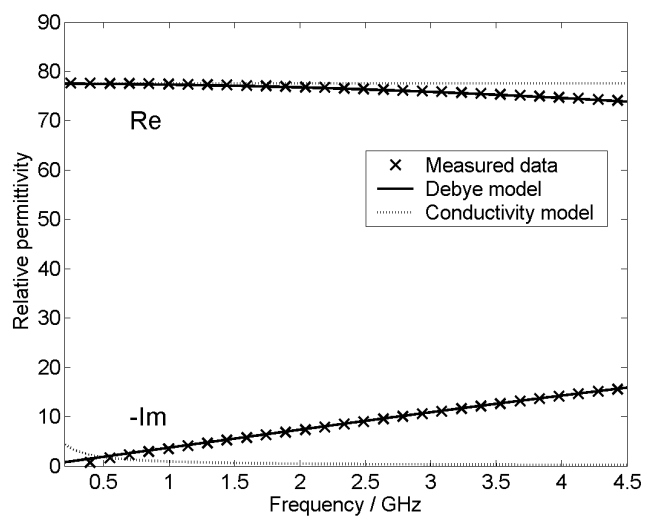

Fig. 5. In this figure the dielectric properties of pure water has been measured. The real and imaginary parts of a fitted Debye model have been plotted together with the corresponding conductivity model corresponding to the Debye model at $0.5 \mathrm{GHz}$.

A numerical 2D FDTD antenna model with 20 antennas placed in a circle with radius $0.1 \mathrm{~m}$ was used. All antennas were used consecutively as transmitters while the remaining 19 antennas acted as receivers. The transmitting antenna was modeled as a hard source whereas the fields were directly sampled at the receiving locations. The forward simulations, to generate synthetic measurement data, were made on a FDTD grid with grid size $1 \mathrm{~mm}$ and terminated with 7 layers of CPML. In Fig. 6 the magnitude of the transmission coefficients $S_{21}, S_{51}$ and $S_{91}$ have been plotted. Furthermore the phase of $\mathrm{S}_{21}$ has been plotted. Data was generated using a sinusoidal signal with frequency $f_{c}=3.0 \mathrm{GHz}$ having a Gaussian amplitude modulation with full width half maximum (FWHM) bandwidth $f_{\mathrm{bw}}=3.0 \mathrm{GHz}$. For comparison scattering data generated with a conductivity model was also plotted. In line with what can be expected the results show good agreement between the two cases around $0.5 \mathrm{GHz}$ but the further away from this frequency the more the results deviate from each other. The effect is relatively more pronounced for the amplitude data than for the phase data, which can be expected since the largest deviation in the conductivity model is seen in the imaginary part. And furthermore the differences in the scattering data between the two models are more significant than the previous case in Fig. 3.

Using synthetic data from the Debye model simulation of the empty system and a corresponding simulation with the test object immersed in the background the image was reconstructed using both the Debye and the conductivity model. A circular area with radius $R_{\mathrm{rd}}=90 \mathrm{~mm}$ centered in the antenna array was reconstructed. The original target object used in this example consisted of several circular objects, each with different radius. For the Debye model the $\epsilon_{\text {static }}$ of the original target has been plotted in Fig. 7 (a). Fig. 7(b) shows 


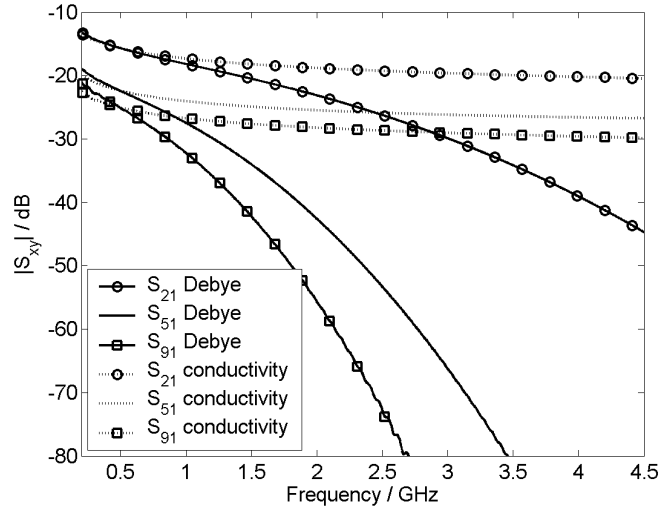

(a) Magnitude

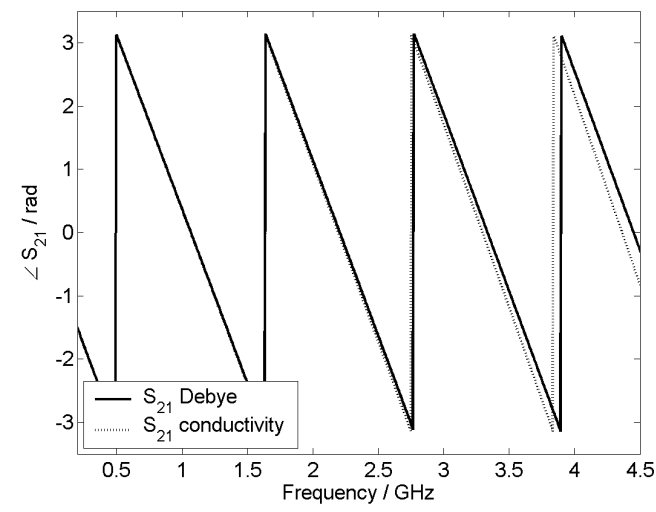

(b) Phase

Fig. 6. Using both Debye model dielectric data and conductivity model data corresponding to the frequency $0.5 \mathrm{GHz}$ scattering data for the background was computed. (a) The magnitude of a few selected transmission coefficients. The solid lines represent the result from the Debye model and the dotted lines the conductivity model. (b) The phase of the transmission coefficient $S_{21}$.

the distribution of $\epsilon_{c}$ of the conductivity model deduced by evaluating the real part of the Debye model at $0.5 \mathrm{GHz}$. Since the sizes of the targets varied to such a large extent it was necessary to use wideband data to resolve the small objects and to beat the non-linearity of the reconstruction problem. Therefore the time domain correspondence of frequency-hopping, [24], has been utilized and the image has been reconstructed in steps using electromagnetic pulses with increasing spectral content. Five iterations were made with each of the center frequencies $f_{c}=0.5,1.0,1.5,2.0,2.5,3.0,3.5,4.0 \mathrm{GHz}$ and FWHM bandwidths $0.5 \mathrm{GHz}$ for $f_{c}=0.5 \mathrm{GHz}$ and 1.0 $\mathrm{GHz}$ for the other center frequencies. In the first iteration at frequency $f_{c}=0.5 \mathrm{GHz}$ the reconstruction was started from an empty background and when moving to a higher frequency the result from the previous pulse was used as a starting point for the forthcoming iterations. This also means that the entire frequency span have contributed to the final images which can be seen in Fig. 7 (k) and (1). Furthermore a number of intermediate reconstructed images in the frequency hopping process have been shown. Here it can be seen how the fine details are refined when more frequency content are added. But also it can be seen how artifacts appear in the reconstructions with the conductivity model.To avoid committing the inverse crime the image was reconstructed on a $2 \mathrm{~mm}$ grid and Gaussian white noise was added resulting in a signal to noise ratio of $40 \mathrm{~dB}$. For the Debye model both $\epsilon_{\text {static }}$ and $\epsilon_{\infty}$ were reconstructed and for the conductivity model both $\epsilon_{c}$ and $\sigma_{c}$ were reconstructed. Reconstructed images of $\epsilon_{\text {static }}$ and $\epsilon_{c}$ have been plotted in the left and right column respectively. With this choice of complex dielectric parameters the sensitivity in the objective functional with respect to the imaginary part is much smaller than to the real part. The reconstruction error is therefore larger for the parameters $\epsilon_{\infty}$ and $\sigma_{c}$. This adds large additional errors in the reconstruction that are not due to the dispersion modeling and therefore we do not show these images here.

As a measure of the accuracy the relative squared errors in each iteration of the reconstruction process have been plotted in Fig. 8. The error was defined according to equation (25) with the integration made over the reconstruction domain $S$ where $r<R_{\mathrm{rd}}$.

$$
\delta=\frac{\int_{S}\left|\epsilon_{\text {original }}-\epsilon_{\text {reconstructed }}\right|^{2} \mathrm{~d} S}{\int_{S}\left|\epsilon_{\text {original }}-\epsilon_{\text {background }}\right|^{2} \mathrm{~d} S}
$$

In this plot it can clearly be seen that the relative error resulting from the conductivity model is always larger than for the Debye model. However the difference is very small in the first 20 iterations. In the following iterations, corresponding to $f_{c}=2.5 \mathrm{GHz}$ and above, the error for the conductivity model starts to increase over the Debye model error. This is also evident in the reconstructed images in Fig. 7 where the appearance of artifacts can be seen in the reconstruction with the conductivity model. These growing artifacts with larger frequency content can also be understood and related to data in Fig. 5 where primarily the deviation between the imaginary parts of the conductivity model and the Debye model introduces an increasing error in the modeling of the background medium. Another property of the frequency-hopping approach can also be seen in the small objects which are not resolved in the beginning of the reconstruction procedure with the low spectral content. Even if it is difficult to see visually in the reconstructed images at central frequencies larger than 2.5 $\mathrm{GHz}$ the relative error in the Debye model reconstruction continues to decrease all the way up to the final iteration. This is clearly not the case for the conductivity model where the relative squared error instead increases after about 20 iterations, see Fig. 8.

\section{CONClusion}

In this paper we have described an algorithm for reconstruction of dielectric Debye model parameters in microwave tomography. This algorithm has many similarities with the conductivity model algorithm that we have used previously, but differs in the way the Debye model gradients and the parameter scaling are computed. We have investigated imaging of targets immersed in a lossy saline solution and ordinary tap water. Both these liquids show strong dispersive behaviors and their dielectric properties are well described by a Debye model. In the saline solution the losses restrict the 


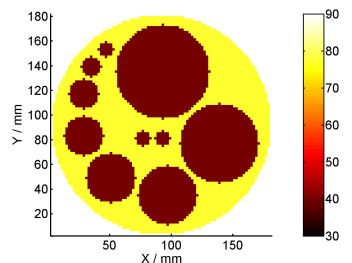

(a) Original $\epsilon_{\text {static }}(\mathbf{x}, \omega)$

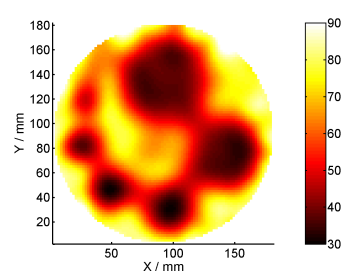

(c) $\epsilon_{\text {static }}, f_{c}=0.5 \mathrm{GHz}$

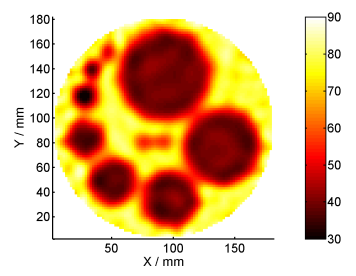

(e) $\epsilon_{\text {static }}, f_{c}=1.0 \mathrm{GHz}$

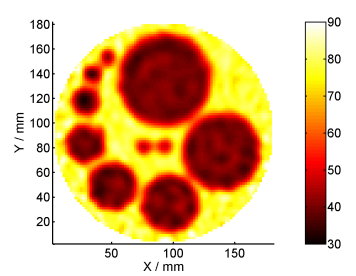

(g) $\epsilon_{\text {static }}, f_{c}=2.0 \mathrm{GHz}$

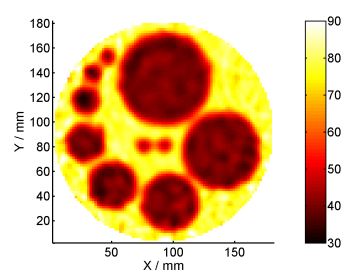

(i) $\epsilon_{\text {static }}, f_{c}=3.0 \mathrm{GHz}$

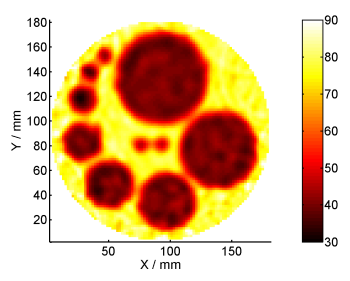

(k) $\epsilon_{\text {static }}, f_{c}=4.0 \mathrm{GHz}$

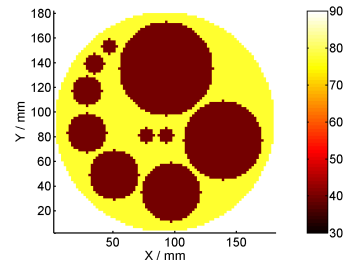

(b) Original $\epsilon_{c}(\mathbf{x}, \omega)$

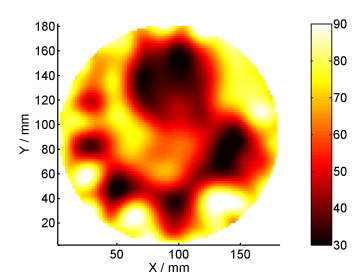

(d) $\epsilon_{c}, f_{c}=0.5 \mathrm{GHz}$

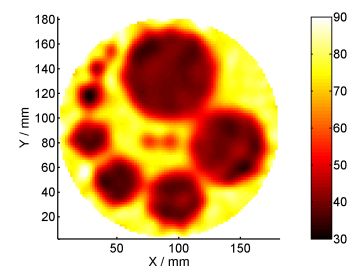

(f) $\epsilon_{c}, f_{c}=1.0 \mathrm{GHz}$

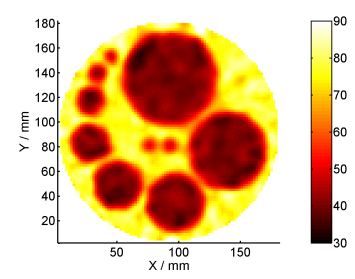

(h) $\epsilon_{c}, f_{c}=2.0 \mathrm{GHz}$

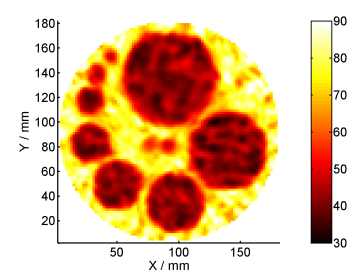

(j) $\epsilon_{c}, f_{c}=3.0 \mathrm{GHz}$

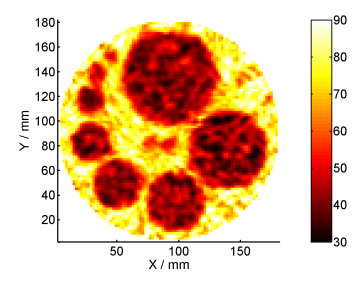

(l) $\epsilon_{c}, f_{c}=4.0 \mathrm{GHz}$
Fig. 7. Reconstructions of the numerical test object. In (a) and (b) the original images have been depicted. In (k) and (l) the final reconstructions using the time domain frequency-hopping approach for the Debye and conductivity models respectively. Also shown are some intermediate steps during the frequency-hopping to the final reconstructions. The center frequency used for the different iterations are given below the image with the FWHM bandwidths for $f_{c}=0.5 \mathrm{GHz}$ set to $0.5 \mathrm{GHz}$ and $1.0 \mathrm{GHz}$ for the other cases. The scale is given in relative permittivity.

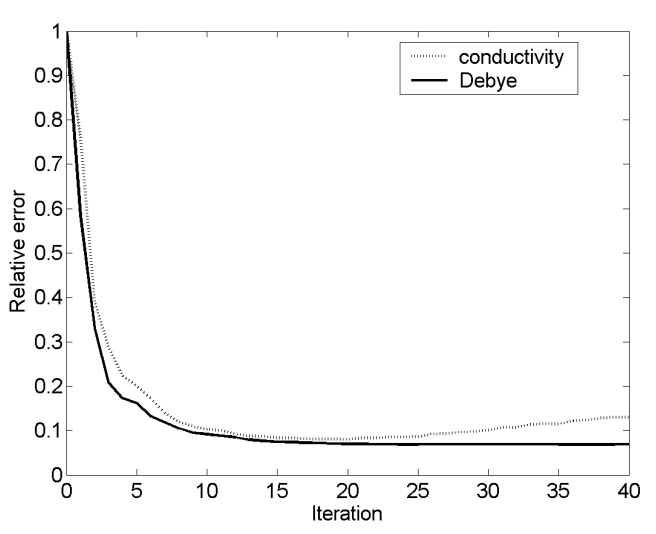

Fig. 8. The relative errors for the Debye model $\epsilon_{\text {static }}$ (solid line) and the conductivity model $\epsilon_{\infty}$ (dotted line) as a function of iteration number.

useful bandwidth for imaging purposes. The deviation from a conductivity model is fairly small but still there is a slight tendency to larger and more artifacts in the reconstructions with the conductivity model. Using the less lossy tap water, which is still strongly dispersive and best described by a Debye model, the useful bandwidth that can be used for imaging is significantly increased. In this case, we can also see a clear advantage in using a reconstruction algorithm based on the Debye model when using wide band data for the imaging. Our results indicate that for a bandwidth of more than about $1.5-2.0 \mathrm{GHz}$ the Debye model starts to give a real and notable improvement in the results.

\section{ACKNOWLEDGMENT}

The computations were performed on the C3SE computing resources.

\section{REFERENCES}

[1] P. M. Meaney, M. W. Fanning, T. Raynolds, C. J. Fox, Q. Fang, C. A. Kogel S. P. Poplack, and K. D. Paulsen, "Initial Clinical Experience with Microwave Breast Imaging in Women with Normal Mammography," Academic Radiology, vol. 14, no. 2, pp. 207-218, Feb. 2007.

[2] S. P. Poplack, T. D. Tosteson, W. A. Wells, B. W. Pogue, P. M. Meaney, A. Hartov, C. A. Kogel, S. K. Soho, J. J. Gibson, and K. D. Paulsen, "Electromagnetic Breast Imaging: Results of a Pilot Study in Women with Abnormal Mammograms," Radiology, vol. 243, no. 2. pp. 350-359, May 2007.

[3] M. Klemm, I. Craddock, J. Leendertz, A. Preece, and R. Benjamin, "Experimental and clinical results of breast cancer detection using UWB microwave radar," in 2008 IEEE Antennas and Propagation Society International Symposium, vol. 1-9, pp. 3014-3017, 2008.

[4] M. Shere, A. Preece, I. Craddock, J. Leendertz, and M. Klemm, "Multistatic radar: first trials of a new breast imaging modality," in Breast Cancer Research, vol. 11, suppl. 2, 2009.

[5] S. S. Chaudhary, R. K. Misha, A. Swarup and J. M. Thomas, "Dielectric properties of normal \& malignant human breast tissue at radiowave and microwave frequencies," Indian J. Biochem. Biophys., vol. 21, pp. 7679, Feb. 1984

[6] W. T. Joines, Y. Zhang, C. Li and R. L. Jirtle, "The measured electrical properties of normal and malignant human tissues from 50 to $900 \mathrm{MHz}$," Med. Phys., vol. 21, pp. 547-550, Apr. 1994.

[7] S. Gabriel, R. W. Lau and C. Gabriel, "The dielectric properties of biological tissues: II. Measurements in the frequency range $10 \mathrm{~Hz}$ to 20 GHz," Phys. Med. Biol., vol. 41, pp. 2251-2269, 1996.

[8] S. Gabriel, R. W. Lau and C. Gabriel, "The dielectric properties of biological tissues: III. Parametric models for the dielectric spectrum of tissues," Phys. Med. Biol., vol. 41, pp. 2271-2293, 1996. 
[9] M. Lazebnik, D. Popovic, L. McCartney, C.B. Watkins, M.J. Lindstrom, J. Harter, S. Sewall, T. Ogilvie, A. Magliocco, T.M. Breslin, W. Temple, D. Mew, J.H. Booske, M. Okoniewski, and S.C. Hagness: "A large-scale study of the ultrawideband microwave dielectric properties of normal, benign, and malignant breast tissues obtained from cancer surgeries," Physics in Medicine and Biology, vol. 52, pp. 6093-6115, Oct. 2007.

[10] M. Lazebnik, C. Zhu, G. M. Palmer, J. Harter, S. Sewall, N. Ramanujam, and S. C. Hagness: "Electromagnetic Spectroscopy of Normal Breast Tissue Specimens Obtained From Reduction Surgeries: Comparison of Optical and Microwave Properties," IEEE Trans. Biomed. Eng., vol. 55, no. 10, pp. 2444-2451, Oct. 2008.

[11] M. Lazebnik, M. Okoniewski, J. H. Booske, and S. C. Hagness: "Highly Accurate Debye Models for Normal and Malignant Breast Tissue Dielectric Properties at Microwave Frequencies," IEEE Microwave and Wireless Component Letters, vol. 17, no. 12, pp. 822-824, Dec. 2007.

[12] A. Taflove and S. C. Hagness, Computational Electrodynamics: The Finite-Difference Time-Domain Method, 3rd ed. Boston, MA: Artech House, 2005.

[13] Q. Fang, P. M. Meaney, and K. D. Paulsen: "Singular Value Analysis of the Jacobian Matrix in Microwave Image Reconstruction," IEEE Trans. Antennas Propag., vol. 54, no. 8, pp.2371-2380, Aug 2006.

[14] B. Brandstätter, K. Hollaus, H. Hutten, M. Mayer, R. Merwa, and H. Scharfetter: "Direct estimation of Cole parameters in multifrequency EIT using a regularized GaussNewton method," Physiological Measurement, vol. 24, pp. 437-448, 2003.

[15] M. Mayer, P. Brunner P, R. Merwa R, F. M. Smolle-Jüttner, A. Maier and $\mathrm{H}$. Scharfetter "Direct reconstruction of tissue parameters from differential multifrequency EIT in vivo," Physiological Measurement, vol. 27, no. 5, pp. S93-S101, 2006.

[16] S. C. Hagness, A. Taflove and J. E. Bridges, "Two-dimensional FDTD analysis of a pulsed microwave confocal system for breast cancer detection: Fixed-focus and antenna-array sensors, IEEE Trans Biomed. Eng., vol. 45, no. 12, pp. 1470-1479, 1998.

[17] P. Kosmas, C. M. Rappaport and E. Bishop. "Modeling With the FDTD Method for Microwave Breast Cancer Detection, IEEE Trans. Microwave Theory Tech., vol. 52, no. 8, pp. 1809-1897, 2004.

[18] Q. Fang, P. M. Meaney, and K. D. Paulsen: "Microwave Image Reconstruction of Tissue Property Dispersion Characteristics Utilizing Multiple-Frequency Information," IEEE Trans. Microwave Theory Tech., vol. 52, no. 8, pp. 1866-1875, Aug. 2004.

[19] D. W. Winters, J. D. Shea, P. Kosmas, B. D. Van Veen, and S. C. Hagness: "Three-Dimensional Microwave Breast Imaging: Dispersive Dielectric Properties Estimation Using Patient-Specific Basis Functions," IEEE Trans. Med. Img., vol. 28, no. 7, pp. 969-981, July 2009.

[20] W. C. Chew and Y. M. Wang: "Reconstruction of Two-Dimensional Permittivity Distribution Using the Distorted Born Iterative Method," IEEE Trans. Med. Img., vol. 9, no. 2, pp. 218-225, June, 1990.

[21] R.F. Remis and P.M. van den Berg, "On the equivalence of the NewtonKantorivich and distorted Born methods, Inverse Problems, vol. 16, no. 1 , pp. 1-4, 2000.

[22] W. C. Chew, Waves and fields in inhomogeneous media, New York: Van Nordstrand Reinhold, 1990.

[23] W. C. Chew and J. H. Lin, "A frequency-hopping approach for microwave imaging of large inhomogeneous bodies," IEEE Microwave Guided Waves Letters, vol. 5, pp. 439-441, Dec., 1995.

[24] A. Fhager, P. Hashemzadeh and M. Persson, "Reconstruction quality and spectral content of an electromagnetic time-domain inversion algorithm," IEEE Trans Biomed. Eng., vol. 53, no. 8, pp. 1594-1604, Aug. 2006.

[25] A. Fhager and M. Persson, "Comparison of two image reconstruction algorithms for microwave tomography," Radio Sci., vol. 40, art.no. RS3017, Jun. 2005.

[26] A. Fhager, S. K. Padhi, and J. Howard, "3D Image Reconstruction in Microwave Tomography Using an Efficient FDTD Model," IEEE Antennas Wirel. Propag. Lett., vol. 8, pp. 1353-1356, 2009.

[27] T. Takenaka, H. Zhou and T. Tanaka, "Inverse scattering for a threedimensional object in the time domain," J. Opt. Soc. Am. A, vol. 20, pp. $1867-1874,2003$.

[28] M. Gustafsson and S. He, "An optimization approach to two-dimensional time domain electromagnetic inverse problems," Radio Sci., vol. 35, no. 2, pp. 525-536, Mar.-Apr. 2000.

[29] I. T. Rekanos, "Time-domain inverse scattering using Lagrange multipliers: an iterative FDTD-based optimization technique," J. Electromagnet. Wave., vol. 17, no. 2, pp. 271-289, 2003.

[30] I. T. Rekanos and A. Räisänen, "Microwave imaging in the time domain of buried multiple scatterers by using an FDTD-based optimization technique," IEEE Trans. Magnetics, vol. 39, pp. 1381-1384. 2003
[31] M. Gustafsson, Ph.D. dissertation, "Wave Splitting in Direct and Inverse Scattering Problems," Lund University, Lund, Sweden, 2000.

[32] A. Tarantola, Inverse Problem Theory, Elsevier Science Publishers, Amsterdam, 1987

[33] S. Nordebo, A. Fhager, M. Gustafsson and M. Persson, "A Systematic Approach to Robust Preconditioning for Gradient-Based Inverse Scattering Algorithms," Inverse Prob., vol. 24, no. 2, art.no. 025027, April, 2008.

[34] S. Nordebo, A. Fhager, M. Gustafsson, B. Nilsson,“ A Green's function approach to Fisher information analysis and preconditioning in microwave tomography," Inverse Problems in Science and Engineering, vol. 18, no. 8, pp. 1043-1063, 2010.

[35] R. M. Mäkinen, J. S. Juntunen, and M. A. Kivikoski, "An improved thin-wire model for FDTD," IEEE Trans. Microw. Theory Tech., vol. 50, pp. 1245-1255, May 2002.

[36] J. Juntunen, "Note on the S-parameter and input impedance extraction in antenna simulations using FDTD," Microw. Opt. Technol. Lett., vol. 28, pp. 8-11, Jan. 2001

[37] O. P. M. Pekonen and J. Xu, "Rigorous analysis of circuit parameter extraction from an FDTD simulation excited with a resistive voltage source," Microw. Opt. Technol. Lett., vol. 12, pp. 205-210, Jul. 1996.

[38] Q. Fang, P. M. Meaney, S. D. Geimer, A. V. Streltsov and K. D. Paulsen, "Microwave image reconstruction from 3D fields coupled to 2D parameter estimation," IEEE Trans. Med. Img., vol. 23, pp. 475-484, Apr. 2004.

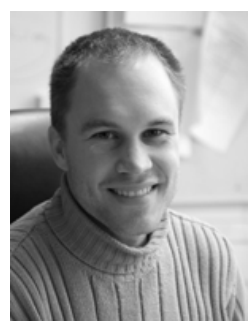

Andreas Fhager received the M.Sc. degree in engineering physics from Chalmers University of Technology, Gteborg, Sweden in 2001. In 2006 he received the $\mathrm{Ph} . \mathrm{D}$. degree in electrical engineering and in 2011 he was appointed Docent in the same field, also at Chalmers. His research interests include electromagnetic imaging methods for breast cancer detection and other biomedical applications of microwaves.

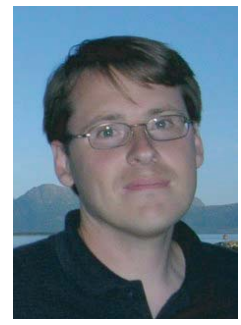

Mats Gustafsson (M'05) received the M.Sc. degree in Engineering Physics in 1994, the Ph.D. degree in Electromagnetic Theory in 2000, and was appointed Docent in Electromagnetic Theory in 2005, all from Lund University, Sweden.

In 2000 he joined the Electromagnetic Theory Group where he is presently a professor. $\mathrm{He}$ cofounded the company Phase holographic imaging $\mathrm{AB}$ in 2004. His research interests are in scattering and antenna theory and inverse scattering and imaging with applications in microwave tomography and digital holography. He has written over 50 peer reviewed journal papers and over 60 conference papers. He received the best antenna poster prize at EuCAP 2007 and the IEEE Schelkunoff Transactions Prize Paper Award 2010.

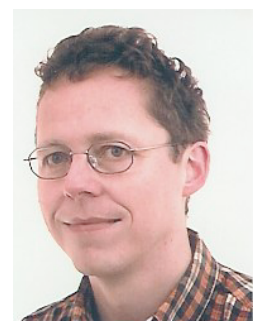

Sven Nordebo received the M.S. degree in electrical engineering from the Royal Institute of Technology, Stockholm, Sweden, in 1989, and the Ph.D. degree in signal processing from Luleå University of Technology, Luleå, Sweden, in 1995 . He was appointed Docent in signal processing 1999. Since 2002 he is a Professor of Signal Processing at the School of Computer Science, Physics and Mathematics, Linnaeus University. Since 2009 he is a Guest Professor of Signal Processing at the Department of Electrical and Information Technology, Lund University. His research interests are in statistical signal processing, sensor array and multichannel processing, wireless communications, antennas and propagation, electromagnetics, inverse problems and imaging, microwave tomography, electrical impedance tomography. 